\title{
Pleural abrasion: a new method of pleurodesis
}

\author{
U U Nkere, S C Griffin, S W Fountain
}

\begin{abstract}
Sixty patients (48 male, 12 female; median age 32 (range 16-72) years) underwent pleural abrasion for persistent or recurrent pneumothorax. Fifty patients had recurrent pneumothorax and 10 persistence of a first pneumothorax despite conservative treatment; two had bilateral pneumothoraces. Pleural abrasion was carried out with a domestic nylon scouring pad and blebs or bullae were ligated or stapled and excised. Intercostal drainage was discontinued after a median time of two days, median serosanguinous loss was $250 \mathrm{ml}$, and the median postoperative stay in hospital was four days. During the median follow up period of 32 (range 1952) months pneumothorax has recurred in one patient.
\end{abstract}

Spontaneous pneumothorax results from the rupture of a subpleural bleb or bulla. The aims of treatment are to alleviate symptoms, obtain full re-expansion of the lung, and prevent recurrence.

Conservative measures, from observation to intercostal drainage alone, have a substantial failure rate and are associated with recurrence in $30-50 \%$ of cases. ${ }^{1}$ Chemical pleurodesis is more effective, but visualisation, which is necessary to achieve good results, is limited even with thoracoscopy. The cause of the problem is not dealt with, and the long term effects of some of the agents used are uncertain. $^{2}$

Thoracotomy provides the opportunity to close the air leak and recurrence is rare after parietal pleurectomy but complications in both the short and the long term are not uncommon. ${ }^{34}$ Pleural abrasion produces a milder inflammatory response, and may minimise the chance of these complications and allow earlier mobilisation and discharge.

Department of Thoracic Surgery, Harefield Hospital, Harefield, Middlesex UB9 6JH

U U Nkere

$S$ C Griffin

$S$ W Fountain

Reprint requests to:

Mr Fountain

Accepted 26 April 1991

\author{
Methods \\ PATIENTS \\ Sixty patients were treated in the course of \\ two years and nine months. Forty eight were \\ men and 12 were women, with a median age \\ of 32 years and a range of 16-72 years (figure). \\ Coexisting respiratory disease was present in \\ four patients (fibrosing alveolitis in one, \\ asthma in one, and clinical chronic obstructive \\ airways disease in two). Nine further patients \\ with spontaneous pneumothorax were con-
}

sidered unfit for surgical treatment because of severe advanced chronic obstructive airways disease.

Ten patients were transferred from another hospital after unsuccessful treatment of a first pneumothorax by intercostal drainage. All had undergone conservative treatment for six days or more. Six patients had had a previous pneumothorax and 44 had had two or more. There were 36 right sided, 22 left sided, and two bilateral pneumothoraces; 26 patients had an intercostal tube in place at the time of surgery, indicating failure of conservative treatment on that occasion.

\section{OPERATIVE TECHNIQUE}

Patients were anaesthetised and ventilated with a double lumen endotracheal tube. In patients with unilateral disease a small (10$12 \mathrm{~cm}$ ) lateral thoracotomy, parallel to the medial border of the scapula, was used, dividing latissimus dorsi but sparing serratus anterior. The chest was entered through the fifth intercostal space. In cases of bilateral disease a midline sternotomy was used. Preexisting adhesions were divided and all blebs or larger bullae were dealt with by ligation or stapling and excision. Where only a scarred area of lung could be identified, indicating fibrosis around a ruptured bleb, this was stapled and excised. The parietal pleura was then abraded with a domestic nylon scouring pad sterilised with ethylene oxide. The mediastinal pleura was spared, but elsewhere abrasion was carried out until capillary oozing appeared. The visceral pleura was also scoured lightly. One intercostal drain was used routinely, but two were used when there was extensive bullous disease or vascular adhesions. Suction was applied to all drains for the first 12-18 hours whether or not there was an air leak to ensure that the lung remained inflated and that the two pleural

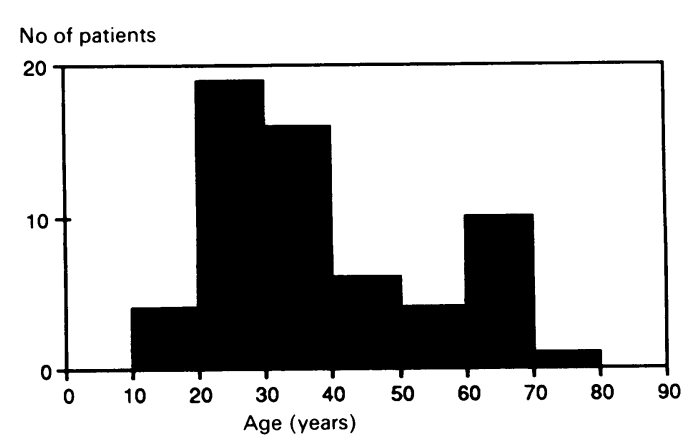

Age distribution of the patients. 
surfaces would remain in contact long enough for adhesions to form.

\section{Results}

Fifty one patients had an identifiable lung abnomality, which was dealt with as described. Of these, $\mathbf{4 5}$ had abnormalities at the apex of an upper lobe only; in three patients the lower lobes only were affected and in one there were blebs at the apices of both lobes. Two cases had only scarring at the apex of the upper lobes. In nine patients no cause for the pneumothorax was found.

Intercostal drainage was required for a median time of two (range 1-11) days but only one patient had an air leak for more than seven days. The median postoperative serosanguinous loss was 250 (range 50$800) \mathrm{ml}$. There were no repeat operations for bleeding but one patient developed a postoperative urinary tract infection and required transfusion for a falling haemoglobin concentration. One patient developed a chest infection and another urinary retention. A pneumothorax occurred in one patient after removal of the intercostal tube; another tube was inserted. The lungs of all patients were fully inflated at the time of discharge. There were no deaths. All patients were pain free and fully mobile at routine follow up six weeks after discharge and none has reported post-thoracotomy pain.

The median follow up period was 32 (range 19-52) months. One patient had a further pneumothorax seven months after treatment, causing collapse of the right lower lobe only. At the second operation a small row of blebs on the superior surface of the middle lobe was seen and stapled. There were patchy avascular adhesions throughout the pleural cavity, which were removed without difficulty by sharp intrapleural dissection so that the lung could be inspected. The abrasion was repeated.

\section{Discussion}

Primary spontaneous pneumothorax occurs predominantly in young healthy adults, mainly in men. In this series the male to female ratio was 4:1. After a fall in incidence with age the rise in patients over 60 is due to the occurrence of pneumothorax as a complication of emphysema and bullous disease. ${ }^{5}$

The indications for active treatment of pneumothorax are not clearcut, but most would agree that one occupying more than $20 \%$ of a hemithorax should be managed by intervention. An advantage of aspiration ${ }^{6}$ over intercostal drainage is that it may be performed as an outpatient procedure. Nevertheless, in the long term non-operative methods of treatment are successful in only $50-70 \%$ of cases. ${ }^{7}$ Chemical pleurodesis carries a higher success rate ${ }^{8}$ but if recurrence does take place further treatment may be made technically difficult by the presence of patchy but dense adhesions. The uncertain long term con- sequences of talc insufflation in young people must also be borne in mind. ${ }^{9}$

Parietal pleurectomy ${ }^{10}$ has the highest success rate of any currently described procedure, but full pleurectomy has been criticised as being too radical. ${ }^{11}$ Early complications, such as haemorrhage requiring reoperation ${ }^{12}$ and Horner's syndrome, ${ }^{13}$ and the development of fibrothorax ${ }^{14}$ have been described. Apical pleurectomy through an axillary incision ${ }^{15}$ has proved equally effective in young patients with minimal lung disease and localised adhesions, but its application is limited to this group. The advantages of pleural abrasion ${ }^{16}$ over a standard pleurectomy relate to the incidence of complications and the dubious logic of treating an abnormality of the lung by removing a large area of normal pleura. Treatment of pneumothorax by closing the air leak alone has been shown to be effective ${ }^{4}$ and careful attention to this is crucial to the success of pleural abrasion. The use of a limited lateral thoracotomy, which can be extended easily if necessary, enables all patients to be treated by the same technique. The pan scrubber we used is cheap and easily available and was sufficiently malleable to reach all parts of the pleural space. The pathological trauma produced by abrasion is mild by comparison with the inflammatory response to pleurectomy and this may explain why recovery was quicker and discharge earlier in our study than in most reports of pleurectomy. ${ }^{12} \mathrm{~A}$ prospective study is needed, however, for the techniques to be properly compared.

The development of thoracoscopic techniques, ${ }^{17}$ possibly combined with the use of the laser, ${ }^{18}$ may well make open surgery for pneumothorax unnecessary in the future. Until these procedures are validated pleural abrasion remains an effective, safe, and simple method for treating spontaneous pneumothorax.

1 Kenneth G, Fischer CR, Gago O, Morris JD, Prager RL The efficacy and timing of operative intervention for spontaneous pneumothorax. Ann Thorac Surg 1986; 42:540-2.

2 Research Committee of the British Thoracic Association and the Medical Research Council Pneumoconiosis Unit. A survey of the long-term effects of talc and kaolin pleurodesis. Br J Dis Chest 1979;73:285-8.

3 Mills M, Baisch BF. Spontaneous pneumothorax: a series of 400 cases. Ann Thorac Surg 1965;1:286-97.

4 Ferguson LJ, Imrie CW, Hutchison J. Excision of bullae without pleurectomy in patients with spontaneous pneumothorax. Br J Surg 1981;68:214-6.

5 Morgan MDL, Edwards CW, Morris J, Matthews HR. Origin and behaviour of emphysematous bullae. Thorax 1989;44:533-8.

6 Bevelaqua FA, Aranda C. Management of spontaneous pneumothorax with small lumen catheter manual aspiration. Chest 1982;81:693-4.

7 Verschoof AC, Ten Velde GPM, Greve LH, Wouters EFM. Thoracoscopic pleurodesis in the management of spontaneous pneumothorax. Respiration 1988;53:197-200.

8 Merete A, Lange P, Viskum K. Spontaneous pneumothorax: comparison of simple drainage, talc pleurodesis, and tetracycline pleurodesis. Thorax 1989;44:627-30.

9 Lange P, Mortensen J, Groth S. Lung function 22-35 years after treatment of idiopathic spontaneous pneumothorax with talc poudrage or simple drainage. Thorax 1988; 43:559-61.

10 Gaensler EA. Parietal pleurectomy for recurrent spontaneous pneumothorax. Surg Gynecol Obstet 1956;102: 293-308. 
11 Clagett OT. The management of spontaneous pneumothorax. J Thorac Cardiovasc Surg 1968;55:761-2.

12 Weeden D, Smith G. Surgical experience in the management of spontaneous pneumothorax. Thorax 1983; 38:737-43.

13 Singh SV. The surgical treatment of spontaneous pneumothorax by parietal pleurectomy. Scand $J$ Thor Cardiovasc Surg 1982;16:75-80.

14 Thomas PA, Gebauer PW. Results and complications of pleurectomy for bullous emphysema and recurren pneumothorax. J Thorac Surg 1960;39:194-201.

15 Deslauriers J, Beaulieu $M$, Despres JP, Lemieux $M$ Leblanc J, Desmeules M. Transaxillary pleurectomy for treatment of spontaneous pneumothorax. Ann Thorac 1980;30:569-74.

16 Youmans CR, Williams RD, McMinn MR, Derrick JR Surgical management of spontaneous pneumothorax by bleb ligation and dry sponge abrasion. Am J Surg 1970;120:644-8.

17 Wakabayashi A. Thoracoscopic ablation of blebs in the treatment of recurrent or persistent spontaneous pneumothorax. Ann Thorac Surg 1989;48:651-3.

18 LoCicero J III, Hartz RS, Frederiksen JW, Michaelis LL. New applications of the laser in pulmonary surgery: hemostasis and sealing of air leaks. Ann Thorac Surg 1985;40:546-50. 